\title{
Effect of Nursing Intervention on Improving the Cognitive Function of Patients with Mild Cognitive Impairment
}

\begin{abstract}
Li Sun*, Zhenzhu Shang
Department of Encephalopathy, Weifang Hospital of Traditional Chinese Medicine, Weifang 261041, Shandong Province, China

*Corresponding author: Li Sun, Sunli1974@126.com

Abstract: Objective: To explore the effect of nursing intervention on improving the cognitive function of patients with mild cognitive impairment. Methods: Sixty patients with mild cognitive impairment in Weifang Hospital of Traditional Chinese Medicine from January 2020 to January 2021 were randomly selected for this study. They were divided into two groups: a reference group (routine follow-up and daily health education) and a research group (nursing intervention based on the reference group). Results: Before nursing, there was no significant difference in the MoCA, MMSE, ADL, SDS, and SAS scores between the two groups $(p>0.05)$. After intervention, the MOCA score and MMSE score of the research group were lower than those of the reference group, the ADL score of the research group was higher than that of the reference group, and the quality-of-life score of the research group was also higher than that of the reference group $(p<0.05)$. Conclusion: Early nursing intervention for patients with mild cognitive impairment can effectively improve their cognitive functions and daily abilities.
\end{abstract}

Keywords: Mild cognitive impairment; Cognitive function; Nursing intervention

Publication date: September 2021; Online publication: September 30, 2021

\section{Introduction}

Mild cognitive impairment mainly refers to the mild impairment of cognitive function caused by various pathological factors. Mild cognitive impairment significantly reduces patients' ability in carrying out activities of daily living and may even lead to the development of Alzheimer's disease, which would have a great impact on their quality of life along with their physical and mental health. Therefore, it is necessary to intervene as soon as possible in order to improve patients' cognitive impairment and their quality of life ${ }^{[1]}$. Based on this, the effect of nursing intervention on improving mild cognitive impairment has been explored.

\section{Material and methods}

\subsection{Basic data}

The subjects that were included in this study were 60 patients with mild cognitive impairment in Weifang Hospital of Traditional Chinese Medicine from January 2020 to January 2021. The inclusion criteria were patients that had met the diagnostic criteria of mild cognitive impairment as well as those that had signed the informed consent form for this study. The exclusion criteria were patients with serious physical diseases, decreased cognitive function due to other mental diseases or factors, as well as those with visual, hearing, or language impairment. The patients with mild cognitive impairment were divided into two groups by the 
digital table method. There were 30 cases in the reference group; the proportion of men to women was 19:11; their age range was between 56 to 74 years old, with an average age of $61.34 \pm 2.56$. There were also 30 cases in the research group; the proportion of men to women was 18:12; their age range was between 57 to 73 years old, with an average age of $61.45 \pm 2.48$. There was no significant difference between the two groups $(p>0.05)$.

\subsection{Methods}

The reference group underwent routine follow-up and daily health guidance whereas the research group received nursing intervention. The nursing intervention for the research group was carried out in different areas.

(1) Life ability nursing

The nurses guided and helped the patients to effectively rebuild their self-care ability. They guided the patients in their daily life activities and actively encouraged them to participate in collective activities, such as Tai Chi and origami, so that the patients were able to come into contact with the environment as much as possible, change their life attitude, and avoid mental decline.

(2) Psychological nursing

The nurses had to quickly gauge the psychological status of the patients, eliminate the loneliness experienced by the patients as much as possible, and provide care to these patients in view of their emotional vulnerability. For patients with language barriers, the nurses had to slow down their speed of speaking and take care of the patients patiently.

(3) Diet nursing

The nurses had to reasonably design personalized diet plans according to the actual situation of the patients and help them to establish a proper nutritional diet structure. They had to ensure that the patients consumed food with higher contents of vitamins and proteins while appropriately adding fruits and vegetables to increase their unsaturated fatty acid levels as well as improve their bodies' condition. These are all conducive for the rapid recovery of the disease.

(4) Intellectual training

In regard to intellectual training, memory training was first conducted. Various trainings were carried out in strict accordance with the theoretical standard of 3R mental stimulation. It was mainly divided into three steps: training of past memories, training of physical positioning, and stimulation training to improve the memory of the patients. Finally, behavior training was conducted to reasonably guide patients to form appropriate behaviors, promote basic common sense to effectively awaken the memory in the patients' brains, and encourage them to memorize behavior to avoid loss.

(5) Social lectures

When the condition of the patients improved to a certain extent, the nurses had to provide family nursing knowledge to the patients and their family members in order to ensure that the patients were able to master basic disease prevention and rehabilitation knowledge, improve and cultivate awareness in regard to prevention and health care, as well as timely carry out supervision and management with the help of social lectures. These are carried out so that when an abnormality occurs, it can be handled promptly to avoid other adverse events.

(6) Regular follow-up

The patients were regularly followed-up for about 15 or 30 days via telephone or door-to-door visits. During the follow-up, the patients were urged to carry out various daily trainings to ensure that there would be an effective improvement in regard to their cognitive functions. 


\subsection{Observation indicators}

The improvement of the cognitive functions of the two groups were observed and compared. The Montreal cognitive assessment (MoCA) was used to evaluate the improvement of cognitive function before nursing, 2 weeks, 4 weeks, and 8 weeks after nursing. The lower the score of the scale, the better the improvement effect of the patients' cognitive dysfunction.

The mini-mental state examination (MMSE) was used to evaluate the intelligence status and cognitive impairment of the patients. The higher the score, the higher the intelligence status of the patients. At the same time, the activity of daily living scale (ADL) was used to evaluate the patients' ability in carrying out activities of daily living. The higher the score, the higher the patients' living ability ${ }^{[2]}$.

The improvement of anxiety and depression in the two groups was evaluated by using Zung's SelfRating Anxiety Scale (SAS) and the Self-Rating Depression Scale (SDS). The dividing value was 50. The higher the score, the more serious the patients' anxiety and depression. The 36-Item Short Form Survey (SF-36) was used to evaluate the improvement of the patients' quality of life. The full score was 100 points with regard to the patients' physiological function, mental health, mental state, and social function. The higher the score, the higher the patients' quality of life ${ }^{[3]}$.

\subsection{Statistical analysis}

Statistical Package for the Social Sciences (SPSS) version 23.0 was used for processing, analysis, and ttest. They were expressed in $(\bar{x} \pm \mathrm{s})$. There is a difference in the data if $p<0.05$.

\section{Results}

\subsection{Improvement of cognitive function}

Comparing the data in Table 1, there was no significant difference in MoCA scores between the two groups before nursing, $p>0.05$. However, after nursing, the MoCA score of the research group was lower than that of the reference group $(p<0.05)$.

Table 1. Comparison of MoCA scores before and after nursing $(\bar{x} \pm \mathrm{s})$ between the two groups

\begin{tabular}{cccccc}
\hline Group & $\mathrm{N}$ (example) & Before nursing & 2 weeks after nursing & 4 weeks after nursing & 8 weeks after nursing \\
\hline Reference group & 30 & $32.25 \pm 3.96$ & $30.87 \pm 3.51$ & $29.09 \pm 3.02$ & $28.25 \pm 2.14$ \\
Research group & 30 & $32.31 \pm 3.85$ & $28.09 \pm 2.12$ & $27.71 \pm 2.09$ & $26.66 \pm 1.95$ \\
$\mathrm{t}$ & & 0.0595 & 3.7133 & 2.0581 & 3.0080 \\
$p$ & & 0.9528 & 0.0005 & 0.0441 & 0.0039 \\
\hline
\end{tabular}

\subsection{Improvement of living ability}

As shown in Table 2, there was no significant difference between the two groups before nursing, $p>0.05$. After nursing, the MMSE and ADL scores of research group were higher than those in the reference group $(p<0.05)$. 
Table 2. Comparison of MMSE and ADL scores $(\bar{x} \pm s)$ before and after nursing between the two groups

\begin{tabular}{cccccc}
\hline \multirow{2}{*}{ Group } & \multirow{2}{*}{ (example) } & \multicolumn{2}{c}{ MMSE } & \multicolumn{2}{c}{ ADL } \\
\cline { 3 - 6 } & & Before nursing & After nursing & Before nursing & After nursing \\
\hline Reference group & 30 & $21.46 \pm 1.58$ & $23.05 \pm 1.16$ & $73.59 \pm 4.52$ & $76.27 \pm 3.59$ \\
Research group & 30 & $21.51 \pm 1.63$ & $25.18 \pm 1.04$ & $73.48 \pm 4.37$ & $80.12 \pm 4.02$ \\
$\mathrm{t}$ & & 0.1206 & 7.4884 & 0.0958 & 3.9125 \\
$p$ & & 0.9044 & 0.0000 & 0.9240 & 0.0002 \\
\hline
\end{tabular}

\subsection{Psychological status}

As shown in Table 3, there was no significant difference in the SAS and SDS scores between the two groups before nursing $(p>0.05)$. After nursing, the SAS and SDS scores of the research group were lower than those of the reference group $(p<0.05)$.

Table 3. Comparison of SAS and SDS scores $(\bar{x} \pm s)$ before and after nursing between the two groups

\begin{tabular}{cccccc}
\hline \multirow{2}{*}{ Group } & \multirow{2}{*}{ (example) } & \multicolumn{2}{c}{ SAS } & \multicolumn{2}{c}{ SDS } \\
\cline { 3 - 6 } & & Before nursing & After nursing & Before nursing & After nursing \\
\hline Reference group & 30 & $50.03 \pm 2.54$ & $41.37 \pm 2.05$ & $50.12 \pm 2.45$ & $40.48 \pm 2.01$ \\
Research group & 30 & $50.11 \pm 2.39$ & $37.64 \pm 1.87$ & $50.34 \pm 2.61$ & $37.58 \pm 1.94$ \\
$\mathrm{t}$ & & 0.1256 & 7.3628 & 0.3366 & 5.6860 \\
$\mathrm{P}$ & & 0.9005 & 0.0000 & 0.7376 & 0.0000 \\
\hline
\end{tabular}

\subsection{Quality of life assessment}

The quality-of-life score of the research group was higher than that of the reference group $(p<0.05)$ (Table 4).

Table 4. Comparison of quality-of-life scores between the two groups $(\bar{x} \pm s)$

\begin{tabular}{cccccc}
\hline Group & N (example) & Physiological function & Mental health & Mental state & Social Function \\
\hline Reference group & 30 & $80.13 \pm 2.56$ & $80.25 \pm 2.61$ & $81.37 \pm 3.16$ & $80.39 \pm 2.85$ \\
Research group & 30 & $85.47 \pm 3.11$ & $86.35 \pm 3.84$ & $86.57 \pm 4.52$ & $86.74 \pm 4.67$ \\
$\mathrm{t}$ & & 7.2611 & 7.1960 & 5.1643 & 6.3573 \\
$\mathrm{P}$ & & 0.0000 & 0.0000 & 0.0000 & 0.0000 \\
\hline
\end{tabular}

\section{Discussion}

Mild cognitive impairment is a common disease. With the continuous extension of the course of disease, it may eventually develop into Alzheimer's disease if there is no timely intervention; thus, having serious impact on the patients' daily life. In regard to that, timely intervention is needed to improve cognitive functions ${ }^{[4]}$.

According to relevant studies, early and timely nursing intervention for patients with mild cognitive impairment can play a significant role. In the process of nursing intervention, it is necessary to guide patients to carry out various daily life activities, help them to effectively rebuild their abilities, improve their mentality, life attitude, and their compliance. Intelligence and cognitive function training can effectively awaken patients' memory as well as improve their memory function and cognitive function. 
Other than that, psychological nursing and dietary guidance can help patients establish good psychological state and dietary structure as well as provide favorable conditions for disease rehabilitation. At the same time, conducting social lectures and educating patients with relevant knowledge and prevention skills provide opportunities for patients to return to the society. Finally, by following-up and supervising these patients, it would be possible to timely gauge the patients' recovery, find out existing problems, and ensure that timely intervention can be carried out for the improvement of patients' cognitive functions ${ }^{[5-8]}$. The results from this study have shown that the improvement effect seen in all the scoring indexes of the research group after nursing was higher than that of the reference group $(p<0.05)$.

In conclusion, nursing intervention for patients with mild cognitive impairment can improve their cognitive functions, reduce adverse psychological emotions, as well as improve their life ability and quality of life.

\section{Disclosure statement}

The authors declare that there is no conflict of interest.

\section{References}

[1] Wang H, Chi Y, Zhang M, et al., 2020, Effect of Game Based Nursing Intervention on Patients with Type 2 Diabetes Mellitus and Mild Cognitive Impairment. Journal of Nursing, (12): 27-30.

[2] Zheng P, Li D, Zheng X, et al., 2019, Effects of Group Puzzle Games on Emotional State and Cognitive Function of Elderly Patients with Mild Cognitive Impairment. Journal of Nursing Management, 19(07): 80-83.

[3] Liu H, Zeng Q, Zhuang X, et al., 2018, Investigation and Analysis of Mild Cognitive Impairment of the Elderly in a Community in Shanghai and Related Intervention Methods and Effects. International Journal of Psychiatry, 45(2): 288-291.

[4] Zou Z, Liu R, Du J, et al., 2019, Systematic Evaluation of Cognitive Intervention Effect of Virtual Reality Technology on Patients with Mild Cognitive Impairment. Chinese Journal of Practical Nursing, 35(8): 622-626.

[5] Chen Y, Liu X, 2019, Clinical Effect of Psychological Nursing Intervention on Patients with Mild Cognitive Impairment. Inner Mongolia Journal of Medicine, 51(7): 872-874.

[6] Zhang J, Xu X, Ding Z, et al., 2018, Application of Hospital Community Family Nursing Intervention Model in Patients with Mild Cognitive Impairment. China Nursing Management, (9): 1230-1235.

[7] Peng S, Chen X, Jiang M, et al., 2020, Application of Transcranial Repeated Acupuncture Intervention and Routine Acupuncture Intervention Combined with Emotional Nursing in Elderly Patients with Mild Cognitive Impairment Secondary to Acute Ischemic Stroke. Sichuan Traditional Chinese Medicine, 38(08): 200-203.

[8] Liu M, Zeng H, Wang X, et al., 2017, Effect of Multimodal Exercise Training on Improving Physical and Cognitive Function of the Elderly with Mild Cognitive Impairment. PLA Journal of Nursing, 34(9): 23-27. 